\title{
REVIEW ARTICLE
}

\section{Hamstring Injury Recurrence and Prevention: A Review}

Noman Ali Khattak ${ }^{1}$, Yumna Ali ${ }^{1}$, Rabiya Akbar ${ }^{2}$, Fatima Khattak ${ }^{3}$, Sufana Ali $^{4}$

\section{ABSTRACT}

Football stands to be the most favorite sport with approximately four billion fans worldwide. Injuries in the game can often be frustrating to the fans, athletes and the club, affecting performance and financial operations. An avoidance curriculum to help cut down risks of such injuries seems obligatory, concerning the socioeconomic and financial repercussions. An issue that is frequently discussed in sports medicine is Hamstring Strain Injuries (HSI) that is widely seen amongst players which could prevent them from engaging in important games. HSI are one of the most frequently occurring injuries in sport representing approximately $12-$ $24 \%$ of all athletic injuries. There is a high prevalence of hamstring strain injuries in many sports, including soccer.

This review consists of summary of hamstring injury causes, prevention and current practices of treatment. It also evaluates a contemporary method that uses whole-body vibration and its benefits to neuromuscular invigoration and defiance exercises.

Key Words: Football, Hamstring Injury, Injury Recurrence, Rehabitation, Sports Technique.

How to cite this: Khattak NA, Ali Y, Akbar R, Khattak F, Ali S. Hamstring Injury Recurrence and Prevention. A Review. Life and Science. 2021; 2(3): 122-128. doi: http://doi.org/10.37185/LnS.1.1.188

This is an Open Access article distributed under the terms of the Creative Commons Attribution License (http://creativecommons.org/licenses/by/4.0), which permits unrestricted use, distribution, and reproduction in any medium, provided the original work is properly cited.

\section{Introduction}

Ishiocrural Muscles, commonly known as the Hamstring Muscles consists of three broad muscles from the posterior part of the thigh, Semimembranosus, Semitendinosus and Bicep Femoris; from medial to lateral, respectively. The extension of hip and knee flexion are the two important functions of the hamstring muscles. The hamstring muscles hinder the knee extension during the Gait Cycle, prior to the heel strike, in order to consume the kinetic energy and protect the hip and knee joints. ${ }^{1}$ The periodic and recurrent cramps

\footnotetext{
${ }^{1,2}$ Faculty of Medicine and Surgery

School of Sports and Exercise Sciences

University of Rome Tor Vergata, Roma, Italy

${ }^{3}$ Department of Gynecology and Obstetrics

Combined Military Hospital, (CMH), Rawalpindi

${ }^{4}$ Department of Oral Maxillofacial Surgery

Foundation University College of Dentistry and Hospital

Islamabad

Correspondence:

Dr. Noman Ali Khattak

Faculty of Medicine and Surgery

School of Sports and Exercise Sciences

University of Rome Tor Vergata, Roma, Italy

E-mail: nomanali.khattak@gmail.com

Funding Source: NIL; Conflict of Interest: NIL

Received: Mar 16, 2021; Revised: Apr 29, 2021

Accepted: May 20, 2021
}

within the posterior compartment of the thigh are known as a Hamstring Strain. It is often seen in athletes, who engage in sports such as rugby, football, cricket or track-events that require constant and rapid short moves of the muscle, similarly hopping or sprinting. The hamstring strain is a relatively wide classification of a condition that can range from a few torn muscle fibers or a partial muscle tear to a more severe, complete tear of the tendon or muscle fibers. Its rehabilitation is considered rather arduous, due to the delayed healing and persistent symptoms

\section{Hamstring and Quadriceps Muscle Work Ratio}

Some experts admit that hamstring injuries can be caused by muscle disproportion, that a person is highly exposed to threats of hamstring injuries when frontal muscles of the thigh, the quadriceps, are strengthened above of the hamstring muscles.

Engaging in sports practices with prior hamstring injury or a pre-injury state may lead to more severe hamstring injuries since muscle require time to heal Records of previous hamstring injuries are vital for the rehabilitation of muscles, as a study states that the risk rate might increase up to six times if the earlier abuses are left untreated. ${ }^{2}$ An Engebretsen et al study based on over five hundred competitive 
footballers under Hamstring Risk Influences revealed that previous records and frequent hamstring strains are the major factors for a definite hamstring injury. ${ }^{3}$, When the quadriceps disproportionately gain more strength than the hamstring muscles, hamstring strains are more likely to occur. This is duly because of the fluctuation of growth between muscles and bones, especially seen amongst women

\section{Powerful Explosive Movements and Hamstring Muscle}

In a study by Jonhagen $S$. et al a comparison was made between eleven sprinters with recent hamstring injuries with nine uninjured runners. The flexibility of the hamstrings and the eccentric and concentric muscle torque were measured in the hamstrings and quadriceps muscles at different angular velocities. Significant differences were observed between the two groups. It was noticed that the sprinters with the hamstring injuries had hamstrings significantly tighter than the uninjured group. Whereas the sprinters who were uninjured had higher eccentric hamstring torque at all angular velocities and also had higher concentric quadriceps and hamstring torque at $30 \mathrm{deg} / \mathrm{sec}$ but at low velocities. Therefore, it was concluded that sprinters with the history of hamstring are weaker in both eccentric and concentric contractions. ${ }^{4}$

Higashihara A. et al conducted a study where full body kinematics and electromyography activation of the long head of right Biceps Femoris and Semitendinosus was recorded over thirteen male sprinters during overground sprinting at maximum effort during late swing phase. The purpose was to investigate the incidence of hamstring strain injury during overground sprinting. It was observed that the peak musculotendon length was coincidental with the peak electromyographic activation in the long head of Biceps Femoris (right) than with that of Semitendinosus muscle. It was observed that the time of peak musculotendon length in the semitendinosus muscle occurred significantly later than their peak electromyographic activation. It was concluded that long head of Biceps Femoris muscle is more prone towards strains and injuries during the late swing phase of sprinting. ${ }^{5}$

\section{Risk Factors}

Strain avoidance in athletes can be a herculean process because of perpetual hazard variations, but it is crucial to treat the risk elements attentively in order to refrain or reduce the chances of such strains. Wellknown diagnosis contributions are; aging, diminished eccentric stability of hamstrings and a prior hamstring injury (his). Various researches have been conducted to demonstrate different types of athletic strains in the hamstring muscle region. However, only two injuries are frequently discussed in sports healthcare. Highspeed running complexity is an injury correlated with the long head of the Bicep Femoris muscle, widely noticed in sprinter athletes and footballers, which bring them to a sudden halt during a run at maximum pace. ${ }^{6}$ Stretching-type injuries as the name suggests, occur due to intense workouts that include superlative stretches which affect the semimembranosus muscle. These injuries can be at super discomfort, leaving one with sharp, unbearable pain. $^{\text {? }}$

\section{Hamstring Stretching Prior to the Play}

Hamstring injuries are trivial amongst footballers but are generally seen in people, who fail to warm-up prior to exercises. Admittedly, the foremost recorded injury in the event of FIFA 2018, was a hamstring strain incurred by a Russian footballer named Dzagoev. It crudely affected his performance throughout the league as well as his teams', as the injury narrowed his durance in the game. Hamstring injuries can cause frustration amongst players and also coaches, as they limit or forbid the time spent on training or even refraining the players from reporting to the games. ${ }^{8}$

\section{Retracted Hamstring}

As discussed often, hamstring strains are caused in sports where a lot of running, powerful accelerations and decelerations are required. These strains are caused when one or more muscles (from the hamstring complex) or tendons are stretched or torn. A pulled hamstring is the less severe type of strain. Whereas, when a hamstring muscle is ruptured, it causes more serious injury that is the "hamstring tear." With the help of advanced diagnostic capabilities, it has been observed that the complete hamstring avulsions from the ischial insertion have caught huge attention. ${ }^{9}$ In a study George Koulouris et al., twenty-one patients were noticed to have proximal tendon injury out of which sixteen were the avulsions. Only four were the distal tendon injuries. It was also noticed that Biceps 
Femoris muscle was the most frequently injured muscle along with Semimembranosus to be the most uncommon. ${ }^{10}$

\section{Pelvic Discomfort and Hamstring Injuries}

In order to determine the consequences of pelvic location and expansion approach on hamstring flexibility, Sullivan MK et al and team conducted a study, despite the conflicting arguments on the subject. The objective of the study was to correlate Static Stretch (SS) and Proprioceptive Neuromuscular Facilitation (PNF) of hamstring expansion practices while retaining two different pelvic states; Anterior Pelvic Tilt (APT) or Posterior Pelvic Tilt (PPT). Two groups were formed consisting of ten members each, to participate in eight sessions of either APT or PPT positions, performing PNF on one leg and SS on the other. Hamstring Flexibility was then examined by flexing the knee with the hip leveled at 90 Degrees. (Active Knee Extension Test AKET). After carefully inspecting the results, with the help of an in and out ANOVA analysis of expansion methods and pelvic leveling, the study affirmed that the APT group showed a serious improvement of hamstring flexibility $(P=0.0375)$, although SS and PNF did not expose a prompt discrepancy. However, the PPT Group failed to depict an advancement in hamstring flexibility with any of the stretching techniques $(P>0.05)$, suggesting that APT posture is vital for the accession of hamstring flexibility than expansion techniques. ${ }^{11}$

\section{Overload}

An inevitable consequence of the loads placed on the human body during any extreme activity causes an injury. In the Olympic games in Beijing, China, it was analyzed that the second most common injury was found out to be the thigh muscle strains. ${ }^{12}$ Various studies involving Union of European Football Associations Champions League footballers reveal the hamstring muscle to be the culprit in various injuries. ${ }^{13}$ Usually, hamstring strains occur at the myotendinous junction while an eccentric load is applied to the muscle. This excessive eccentric load on the muscle tears the junction between muscle fibers and the musculotendinous junction. The mechanism of eccentric muscle contraction occurs in such a way that during the passive stretching force at the muscle-tendon unit, the force of active contraction is added which puts the muscle at risk of disruption. Usually during the period of eccentric muscle contraction, there is a sudden onset of localized pain. Few of the predisposing risk factors to this type of indirect muscle injury are age, male sex, fatigue previous hamstring strains, etc. ${ }^{14}$

\section{Injury Caused while Sprinting}

Activities that require intense stretching (such as sprinting) hold greater chances of causing a hamstring injury. Therefore, it is essential to identify athletes who are susceptible to a hamstring injury. For such purpose, Yeung SS et al ${ }^{15}$ conducted a study on forty-four sprinters from The Hong Kong Sports Institute, The Hong Kong Amateur Athletic Association and from the intercollegiate athletic teams. The purpose was to investigate the incidence of hamstring muscle injury in an athletic season and to explore possible predictor of this injury. Prior to the athletic season, an assessment was done in order to obtain the hamstring flexibility, concentric and eccentric isokinetic peak torque and peak torque angle. Athletes were kept under observation for over a year and were asked to report in case of an occurrence of an injury during training or competition. It was found out that eight of the athletes had already sustained the hamstring injuries over the season. It was also noticed that the incidence of these injuries was higher at the beginning of the season. This study revealed one more interesting fact that the athletes with the hamstring-quadriceps peak torque ratio less than 0.6 at an angular velocity of $180 \mathrm{deg} / \mathrm{s}$ were at increased risk of a hamstring injury. This study suggested that such assessments can be helpful in identifying the risk of hamstring injuries in the sprinters or other athletes sprinting. ${ }^{15}$

\section{Hamstring Injury Rehabilitation and Recurrence Prevention}

The fundamental objective of the treatment is to assist the athlete to his previous state of health prior to the injury, in other words, to profess the functional rehabilitation. Many studies have been done to accumulate convenient training programs for footballers, to avert hamstring injuries, to ensure avoidance of post-rehab strains contemplating the accurate return to a sport time period or to limit the frequency of hamstring injuries as a whole.

Lee JW et al, ${ }^{16}$ study involved 169 qualified football players, with aim to foresee the repercussion of 
isokinetic durability measures on prospective hamstring injuries. The participants were screened for isokinetic energy measures during pre-season who were then observed throughout a competitive season that lasted for about ten months.

The analytic obligations comprised of concentric conduction of knee flexion and extension respectively at 60 degs-1 / 240 degs- 1 and eccentric performance at 30 degs-1. The study also gauged shortfall of energy, bilateral discrepancies, and hamstring to quadriceps power proportions. Further proved, that a higher threat of acute HSI was identified, in the efficient football players with momentous lower isokinetic hamstring durability, the lower scale of hamstring-to-quadriceps strength and any earlier incidents of Hamstring injuries. ${ }^{16}$

\section{Adverse Neural Tension}

The repetition of Hamstring strains and the root to it are often analyzed through different aspects of studies. And one of those considerations were brought forward by Turl SE and colleagues, they established that 'Adverse Neural Tension' to be one of the crucial scientific components that sources recurrence of Hamstring strains. Adverse Neural Tension is anomalous physiological feedback, caused by impediment within the neural tissue. Adverse Neural Tension is bred by the complexity of the nervous system when its regular spectrum of operations and span efficiency are inspected. ${ }^{17}$

\section{Sports Techniques}

The key to improving flexibility is a proper stretching program. In order to understand, which stretching technique (active or passive) would produce and maintain the greatest gain in hamstring flexibility, a randomized control trial was conducted by MeroniR et al ${ }^{18}$ on sixty-five volunteer healthy subjects. A sixweek stretching program was conducted where these participants were divided into two groups. Group one performed the active stretching exercises. Whereas, the second group performed the passive stretching exercises. Results showed that the participants who performed the active stretching exercises produced that greater gain in active knee extension range of motion and it was also observed that the gain was maintained for four weeks after the training ended. Contrary to this, the passive stretching group was not seen to produce and maintain the gain like that of the active stretching group. Therefore, active stretching was concluded to 125 be more time efficient and needed lower compliance to produce effects on flexibility. ${ }^{18}$

\section{Exercise Recommendations}

\section{The Nordic Hamstring Exercise}

In order to decrease the rate of hamstring injuries, a number of training methods have evolved.

But mainly literature shows that in various sports, during a competitive season, only eccentric exercises have shown to reduce the number of hamstring injuries up to $70 \%{ }^{19}$

To avert a possible hamstring strain during a fatal rhythmic fluctuation of sprinting, it is advised that the eccentric tenacity of the hamstrings is escalated that will facilitate the heightened tension within. The Nordic Hamstring Exercise (NHE) is a structure of eccentric training that helps bolster the hamstrings. This particular exercise being able to probe the precise injury structure is believed to greatly help lessen the HSI than the classic concentric curls. ${ }^{20}$

A team led by Van Der Horst $\mathrm{N}$ strategized a study on a small group of amateur male footballers chosen into two different groups; (intervention and control), to examine the competence of the NHE basing on frequency and severity of hamstring injuries. The intervention group was advised to implement twenty-five huddles of NHE throughout the study and the control group went on about basic football practices. The research lasted for about thirteen weeks and during the 2013 calendar annum, constant hamstring injury incidence and severity were tracked. The data were then collected basing on the criteria primarily on personal attributes such as age, history of strains and field stand provided by a survey and secondarily from injury harshness and consent of intervention pact. Evidently, the results favored the intervention group established steep exposure towards hamstring injuries than the closed group, although injury severity readings did not showcase significant discrepancy between the groups. The study concluded that regular amateur training following the NHE covenant, achieves a greater reduction of Hamstring Injury Incidence, although severity resulting in zero discrepancies. ${ }^{21}$

\section{Static Stretching}

Many studies proposed static stretching techniques for hamstring muscle improves flexibility of the muscle and consider this type of exercises beneficial for the rehabilitation of HSI.

A total of 69 subjects, with a mean age of $16.45 \pm 0.96$ 
years and with limited hamstring flexibility (defined as $20^{\circ}$ loss of knee extension measured with the thigh held at $90^{\circ}$ of hip flexion) were recruited for this study. Differences were significant for test and for the test-by-group interaction. Follow-up analysis indicated significant differences between the control group (gain $=1.67^{\circ}$ ) and both the eccentric-training $\left(\right.$ gain $\left.=12.79^{\circ}\right)$ and static-stretching $\left(\right.$ gain $\left.=12.05^{\circ}\right)$ groups. No difference was found between the eccentric and static-stretching groups. The gains achieved in range of motion of knee extension (indicating improvement in hamstring flexibility) with eccentric training were equal to those made by statically stretching the hamstring muscles. ${ }^{22}$

\section{Targeting Muscle Group for Improvement}

Almost $80 \%$ of HSIs involve the biceps femoris (BF; longhead) muscle and most injuries are thought to occur during the late swing phase of high-speed running. During this phase of the gait cycle, the biceps femoris (BF; longhead) reaches its peak length and develops maximal force while undergoing a powerful eccentric contraction to decelerate the shank forefoot strike, and this may, at least partly, explain its propensity for injury. Prior biceps femoris (BF; longhead) injury is associated with a degree of neuromuscular inhibition and prolonged atrophy, which suggests that current rehabilitation practices do not adequately restore function to this muscle. Hamstring weakness is a risk factor for future strain injury and interventions aimed at increasing strength, particularly eccentric knee flexor strength, have reduced HSI rates in several sports. However, despite an increased focus on hamstring strength in injury preventive programs, exercise prescription in the clinic often does not always rely on empirical evidence. There is currently a very small body of work on the activation patterns of the hamstrings during commonly employed exercises. This area of work can improve the rehabilitation of hamstring injury. ${ }^{23}$

\section{Plyometrics}

Plyometric exercises are explosive movements that demand a high amount of power, agility, and concentration. These exercises help in training the muscles to contract strongly and quickly. These exercises include lunge jump, box jump, hurdles, and Butt kicks. $^{24}$

One study suggested plyometric exercises combined with weight lifting as a strength training program to be highly effective in improving the kicking performance especially for sports like football. ${ }^{25}$ Another study suggested, these plyometric exercises are important in the prevention of injuries by means of improving landing mechanics, decreasing ground reaction forces and by improving hamstring to quadriceps ratio. ${ }^{26}$

\section{Hard Surface Compared to Plyometrics on Trampoline}

The recent studies in this domain many authors have argued about the positive and negative effects of surface used in plyometric exercises in injury prevention programs. Akasaka $\mathrm{K}$ et al suggests that knee extensor moments are primarily a result of quadriceps muscular activity, and in jumping are in response to knee flexion that occurs during landing. This indicates that drop vertical jump (DVJ) landing induces an eccentric contraction of knee extensor muscles, including the quadriceps. The results from this study indicate that DVJ landing after repeated trampoline jumping increased knee extensor moments more than when jumping on the ground. Increased knee extensor moments, associated with trampoline jumping, may be the result of an attempt to increase stability of the knee joint to compensate for the unstable surface on the trampoline. This result indicates that repeated trampoline jumping could have a negative effect of increasing the knee joint stress during landing compared with repeated jumping on the ground. ${ }^{27}$

\section{Whole Body Vibration}

Annino $\mathrm{G}$ et al conducted a study to investigate the neuromuscular activity after the exposure of whole body vibration for ten minutes. Subjects for this study were twenty young male adults who were randomized in two groups. The two groups were the vibration group and the non-vibrated group. The vibration group was exposed to whole-body vibration at $35 \mathrm{~Hz}$ for ten minutes whereas the nonvibrated group was a placebo group, who were not exposed to any vibration. Then subjects from both groups were then evaluated with countermovement jump and muscular flexibility by means of electromyographic analysis recorded: Vastus Lateralis, Vastus Medialis, Biceps Femoris and Gastrocnemius Lateralis. ${ }^{28}$

\section{Rehabilitative Program}

The imperative objectives of Hamstring rehabilitation are to obtain the standard 
performance level ascertained before the injury and to firmly assure the minimize risk of reoccurrence that motivates a safer return to sport practice. ${ }^{29}$

It is essential to keep track of the potentiality and strength of muscles, motion scope, rigidity, and resilience when it comes to exercise science. Alternatively, it is also crucial for the recovery of sports injuries that the neuromuscular performance is monitored regularly. It is also notable that various approaches, trials, and means of auditing such performances are available to record and analyze the functionalities that could help the process of rehabilitation from sports injuries effectively. ${ }^{30} \mathrm{~A}$ vertical squat jump is performed with knees tilted at 90 o degrees and hands comforted by the hip. Similarly, a counter-movement jump starts at a standing position, gradually shifting to a squat jump. A drop jump, on the other hand, is executed from five standard drop heights, such as $20 \mathrm{~cm}, 40 \mathrm{~cm}, 60 \mathrm{~cm}$, $80 \mathrm{~cm}$ and $100 \mathrm{~cm}$; where and athlete drops from the given height and jumps immediately with the hands resting on the hips. ${ }^{31}$

Gabriel Amorim Ramos carried a research, in order to review the present approaches on physical therapy and rehabilitation of hamstring strains to circumvent the persistence. The study included the trials of various systems in use such as cryotherapy, therapeutic ultrasound, moderated laser therapy, labored remedy and curative exercises. ${ }^{32}$

\section{Progression Return to Play Criteria}

Studies reveal that approximately one-third of hamstring injuries persist within a year ensuring the return to sport, believed to be more austere than the initial trauma. Such immense recurrence rate of injuries indicates athletes' untimely return to sports basing on substandard criteria administered by the medical staff. The additional aspects that are contemplated to assist the higher rates of intermittent cramps generally are, enduring deficiency in the injured muscles, residual scar tissue causing shortened extensibility of the musculotendinous unit or the flexible transitions in the biomechanics and motor patterns of sporting operations, following the primary damage. ${ }^{33}$

Athletes' muscle durability and Range of Motion are overseen by the Physical therapists and other health care professionals by manual trials or with the help of handheld dynamometers, following the rehabilitation. However, this still has not improved the repetition of hamstring injuries rather the recurrence rate remaining at highest stakes.

\section{Discussion}

It is firmly acknowledgeable that hamstring injuries are commonplace amongst footballers. ${ }^{3-5}$ Hamstring strain injuries are one of the most common reasons for loss of playing time in athletes at all levels of competition. A comprehensive evaluation for accurate diagnosis and planning of effective rehabilitation program that promotes muscle tissue and functional recovery, is essential to minimize the risk of re-injury and optimize athlete performance. ${ }^{21,25}$ Without adequate rehabilitation, athletes may experience persistent weakness in the injured muscle and adaptive changes in the biomechanics and motor patterns of sporting movements. There is mounting evidence that rehabilitation strategies incorporating neuromuscular control; progressive agility and trunk stabilization; and eccentric strength training are more effective at promoting return to sport and minimize the risk of re-injury. ${ }^{16}$ Dynamic clinical and functional tests can be used to assess readiness for return to sport; however, an athlete should continue independent rehabilitation after return to sport to aid in minimizing re-injury risk. ${ }^{30-32}$

Various factors decide a possible hamstring injury such as maturity, inequality within muscle power, lowered eccentric strength, weakened resilience, neural tension, muscle deficiency, and strain recurrence; the trivial aspect being the previous hamstring injury records. ${ }^{15-17}$

Furthermore, observations proved that stretching before a game or practices are vital in order to counter possible hamstring strain threats. It is crucial that a player is rehabilitated before they are allowed to return to their sport, stating that a poorly treated hamstring injury may cost the player missing important games as chances of hamstring strains reoccurring are higher. ${ }^{20}$ Researchers have also depicted that however, grade I and II hamstring strains have shown a greater effect of healing through whole body vibration rehabilitation practices.

\section{REFERENCES}

1. Linklater JM, Hamilton B, Carmichael J. Hamstring injuries: 
anatomy, imaging, and intervention. Semin Musculoskelet Radiol. 2010; 14: 131-61.

2. Hägglund $M$, Waldén $M$, Ekstrand J. Previous injury as a risk factor for injury in elite football: a prospective study over two consecutive seasons. Br J Sports Med. 2006; 40: 767-72.

3. Engebretsen AH, Myklebust $\mathrm{G}$, Holme I. Intrinsic risk factors for hamstring injuries among male soccer players: a prospective cohort study. Am J Sports Med. 2010; 38: 114753.

4. Jonhagen S, Nemeth G, Eriksson E. Hamstring injuries in sprinters: the role of concentric and eccentric hamstring muscle strength and flexibility. Am J Sports Med. 1994; 22: 262-6.

5. Higashihara A, Nagano $\mathrm{Y}$, Ono T. Relationship between the peak time of hamstring stretch and activation during sprinting. Eur J Sport Sci. 2016; 16: 36-41.

6. Askling $\mathrm{CM}$, Tengvar $\mathrm{M}$, Saartok $\mathrm{T}$. Acute first-time hamstring strains during high-speed running: a longitudinal study including clinical and magnetic resonance imaging findings. Am J Sports Med. 2007; 35: 197-206.

7. Askling $C M$, Tengvar $M$, Saartok T. Acute first-time hamstring strains during slow-speed stretching: clinical, magnetic resonance imaging, and recovery characteristics. Am J Sports Med. 2007; 35: 1716-24.

8. ESPN. Russia's Alan Dzagoev likely to miss rest of World Cup - club doctor. 2018. [updated on: June 2018, accessed on: May 2020]. [available at: https://www.espn.in/football/ russia/story/3526090/russias-alan-dzagoev-set-to-missrest-of-world-cup-with-hamstring-injury]

9. Colosimo AJ, Wyatt HM, Frank KA. Hamstring avulsion injuries. Oper Tech Sports Med. 2005; 13: 80-8.

10. Koulouris $\mathrm{G}$, Connell D. Evaluation of the hamstring muscle complex following acute injury. SkeletRadiol. 2003; 32: 5829.

11. Sullivan MK, Dejulia JJ, Worrell TW. Effect of pelvic position and stretching method on hamstring muscle flexibility. Med Sci Sports Exerc. 1992; 24: 1383-9.

12. Junge $A$, Engebretsen $L$, Mountjoy $M L$, Alonso JM, Renström PA, Aubry MJ, et al. Sports injuries during the summer Olympic Games 2008. Am J Sports Med. 2009; 37: 2165-72.

13. Waldén $M$, Hägglund $M$, Ekstrand J. UEFA Champions League study: a prospective study of injuries in professional football during the 2001-2002 season. Br J Sports Med. 2005 ; 39: 542-6.

14. Lee JC, Mitchell AW, Healy JC. Imaging of muscle injury in the elite athlete. Br J Sports Med. 2012; 85: 1173-85.

15. Yeung SS, Suen AM, Yeung EW. A prospective cohort study of hamstring injuries in competitive sprinters: preseason muscle imbalance as a possible risk factor. Br J Sports Med. 2009; 43: 589-94.

16. Lee JW, Mok KM, Chan HC. Eccentric hamstring strength deficit and poor hamstring-to-quadriceps ratio are risk factors for hamstring strain injury in football: A prospective study of 146 professional players. J Sci Med Sport. 2018; 21: 789-93.

17. Turl SE, George KP. Adverse neural tension: a factor in repetitive hamstring strain? J Orthop Sports Phys Ther. 1998; 27: 16-21.

18. Meroni R, Cerri CG, Lanzarini C, Barindelli G, Della Morte G, Gessaga $V$, et al. Comparison of active stretching technique and static stretching technique on hamstring flexibility. Clin J Sport Med. 2010; 20: 8-14.

19. Arnason A, Andersen TE, Holme I. Prevention of hamstring strains in elite soccer: an intervention study. Scand J Med SciSpor. 2008; 18: 40-8.

20. Mjølsnes R, Arnason A, Østhagen T. A 10-week randomized trial comparing eccentric vs. concentric hamstring strength training in well-trained soccer players. Scand J Med SciSpor. 2004; 14: 311-7.

21. van der Horst N, Smits DW, Petersen J, Goedhart EA, Backx FJ. The preventive effect of the nordic hamstring exercise on hamstring injuries in amateur soccer players: a randomized controlled trial. Am J Sports Med. 2015; 43: 1316-23.

22. Nelson RT, Bandy WD. Eccentric training and static stretching improve hamstring flexibility of high school males. J Athl Train. 2004; 39: 254.

23. Bourne MN, Timmins RG, Opar DA, Pizzari T, Ruddy JD, Sims $C$, et al. An evidence-based framework for strengthening exercises to prevent hamstring injury. Sports Med. 2018 ; 48: 251-67.

24. Radcliffe J, Farentinos R. High-Powered Plyometrics, 2E. $1^{\text {st }}$ Edition. Human Kinetics; 2015

25. Perez-Gomez J, Olmedillas $H$, Delgado-Guerra S, Royo IA, Vicente-Rodriguez G, Ortiz RA, et al. Effects of weight lifting training combined with plyometric exercises on physical fitness, body composition, and knee extension velocity during kicking in football. ApplPhysiolNutr Me. 2008; 33: 501-10.

26. Booth MA, Orr R. Effects of plyometric training on sports performance. J. Strength Cond. Res. 2016; 38: 30-7.

27. Akasaka K, Tamura A, Katsuta A, Sagawa A, Otsudo T, Okubo $Y$, et al. Does trampoline or hard surface jumping influence lower extremity alignment?.J Phys Ther. 2017; 29: 2147-50.

28. Annino $G$, lellamo F, Palazzo $F$, Fusco A, Lombardo $M$, Campoli $F$, et al. Acute changes in neuromuscular activity in vertical jump and flexibility after exposure to whole body vibration. Medicine. 2017; 96: e7629.

29. Heiderscheit BC, Sherry MA, Silder A, Chumanov ES, Thelen DG. Hamstring strain injuries: recommendations for diagnosis, rehabilitation, and injury prevention. J Orthop Sports Phys Ther. 2010; 40: 67-81.

30. Bosco $\mathrm{C}$. Methods of functional testing during rehabilitation exercises. InRehabilitation of Sports Injuries 2001 (pp. 1122). $1^{\text {st }}$ Edition. Springer, Berlin, Heidelberg.

31. Neumann DA. Kinesiology of the hip: a focus on muscular actions. J Orthop Sports Phys Ther. 2010; 40: 82-94.

32. Ramos GA, Arliani GG, Astur DC, Pochini AD, Ejnisman B, Cohen $\mathrm{M}$, et al. Rehabilitation of hamstring muscle injuries: a literature review. Rev Bras Ortop. 2017; 52: 11-6.

33. Erickson LN, Sherry MA. Rehabilitation and return to sport after hamstring strain injury. Sport Health Sci. 2017; 6: 26270 . 